\title{
Resistance of nanofill and nanohybrid resin composites to toothbrush abrasion with calcium carbonate slurry
}

\author{
Toshimitsu SUZUKI ${ }^{1}$, Hideaki KYOIZUMI ${ }^{1}$, Werner J. FINGER ${ }^{2}$, Masafumi KANEHIRA², Tatsuo ENDO², \\ Andreas UTTERODT ${ }^{3}$, Hisashi HISAMITSU ${ }^{1}$ and Masashi KOMATSU ${ }^{2}$ \\ ${ }^{1}$ Department of Clinical Cariology, Showa University School of Dentistry, 2-1-1 Kitasenzoku, Ohta-ku, Tokyo 145-8515, Japan \\ ${ }^{2}$ Division of Operative Dentistry, Department of Restorative Dentistry, Tohoku University Graduate School of Dentistry, 4-1 Seiryo-machi, Aoba-ku, \\ Sendai 980-8575, Japan \\ ${ }^{3}$ Heraeus Kulzer GmbH, Philipp-Reis-Str. 8/13, 61273 Wehrheim, Germany \\ Corresponding author, Toshimitsu SUZUKI; E-mail: suzubin@dent.showa-u.ac.jp
}

\begin{abstract}
The aim of this study was to investigate the wear of four nanofilled resin composites using simulated toothbrushing for 50,000 cycles with calcium carbonate slurry. The depth of abrasion and roughness (Ra) were measured after each 10,000 brushing cycle. The surface texture of the worn samples was examined by SEM.

The wear depths of the nanofill Filtek Supreme XT (FIL), the nanohybrides Grandio (GRA), Tetric EvoCeram (TET), and Venus Diamond (VED) increased linearly with numbers of brushing cycles or approximately 80, 12, 600, and $60 \mu \mathrm{m}$, respectively after 50,000 strokes. Surface roughness showed virtually no change between 10,000 and 50,000 brushing cycles; the ranking order was TET < FIL $<$ GRA < VED. FIL showed rather uniform abrasion with nanoclusters protruding from the surface. TET was very smoothly abraded without signs of debonding of the prepolymerized particles, whereas GRA and VED showed pronounced wear of the matrix polymer surrounding larger glass filler particles.
\end{abstract}

Keywords: Nanofill resin composite, Toothbrush abrasion, Surface roughness

\section{INTRODUCTION}

The term "nanotechnology" has evolved over the years via terminology drift to mean "anything smaller than microtechnology," such as nano powders, and other things that are nanoscale in size, but not referring to mechanisms that have been purposefully built from nanoscale components. This evolved version of the term is more properly labeled "nanoscale bulk technology," while the original meaning is more properly labeled "molecular nanotechnology."

Classical resin composite materials comprise hybrid types containing blends of microscopic $(1-5 \mu \mathrm{m})$ and submicroscopic $(0.4-0.8 \mu \mathrm{m})$ glass particles, and microfill materials, typically containing silica particles (0.04-0.05 $\mu \mathrm{m})$ mostly added in prepolymerized fillers. Based on the definition "nanoscale bulk technology" new classes of resin composite restorative materials, so-called nanocomposites have been developed and marketed during recent years. Such materials are available as nanofill types, containing both discrete nanomer and nanocluster particles, and as nanohybrid compounds containing milled glass fillers and discrete nanoparticles $(40-50 \mathrm{~nm})^{1-3)}$. The reduction in particle size with such new resin composites has apparently opened a new gateway in restorative dentistry. Nanocomposites are claimed to combine the good mechanical strength ${ }^{4-6)}$ of the hybrids and the superior polish of the microfills ${ }^{7}$. Other positive features are reportedly high wear resistance ${ }^{8,9)}$, improved optical characteristics $^{5}$, and reduced polymerization shrinkage ${ }^{4,10)}$.

It is important to evaluate the performance of new restorative materials as a consequence of toothbrushing that may cause loss of material and increased surface roughness. For this purpose a number of wear testing machines simulating toothbrushing mostly with commercial toothpastes have been described and used for testing of the wear resistance of restorative materials ${ }^{11-17)}$. Although the first nanocomposite resins were introduced into dental practice several years ago, there is not much information available on their resistance to toothbrushing wear ${ }^{3,18)}$.

Therefore, the present study evaluated the effects of toothbrushing with calcium carbonate slurry on abrasive wear, surface roughness, and surface texture of one nanofill and three nanohybrid composites using a device for simulated toothbrushing, a 2-D profilometer, and scanning electron microscopy. Additionally, the Knoop Hardness (KHN) was determined of specimens prior to wear testing. The null hypothesis tested was that there would be no differences in wear, surface roughness and texture, and Knoop Hardness between the resin composites tested.

\section{MATERIALS AND METHODS}

The four resin composites used are shown in Table 1 together with their compositions. The applied filler concepts used in these products are different. According to the manufacturer nanofill Filtek Supreme XT (FIL) contains a combination of silica and zirconia nanofiller particles. Grandio (GRA) and Venus Diamond (VED) are nanohybrid composite types. GRA contains fluorosilicate glass, $\mathrm{SiO}_{2}$ microfiller, and spherical nanofiller. VED contains Ba-Al-F-Borosilicate glass and 
$\mathrm{SiO}_{2}$ nanofiller particles. In Tetric EvoCeram (TET) a mixture of glasses, $1 \mu \mathrm{m}$ and 0.4 to $0.7 \mu \mathrm{m}$, and nanofiller in prepolymerized fillers is used.

\section{Specimen preparation}

Resin composite plates were produced in rectangular Teflon molds $(12 \times 3 \times 3 \mathrm{~mm})$ placed on Mylar strip covered glass slides. The molds were bulk filled with slight excess, covered with another Mylar strip and pressed flush with a top glass slide. Each specimen was light activated for 60 seconds with Translux Power Blue (LED Light, output: $650 \mathrm{~mW} / \mathrm{cm}^{2}$, Heraeus Kulzer GmbH, Hanau, Germany) in a slow scanning mode along the long axis of the sample (15 cycles) with the light-emitting window in contact with the strip-covered topside of the specimens. The cured resin composite beams were pushed out of the molds and immediately immersed in deionized water for 24 hours before testing.

\section{Toothbrush abrasion testing}

From each of the 4 resin composites 20 specimens were produced and divided into two groups with equal numbers of specimens. After rinsing and air-drying the ten specimens of each composite material and each group were mounted with the light-activated side up using double-faced adhesive tape on an acrylic resin plate $(48 \times 10 \times 3 \mathrm{~mm})$ next and in close contact to each other's $3 \mathrm{~mm}$ high and $12 \mathrm{~mm}$ long surfaces. Next to the ten resin composite specimens three acrylic resin samples of the same size were mounted at each side. In the first group the target surfaces of the mounted specimens were slightly ground on wet $\mathrm{SiC}$ paper, grit \#600 for 60 seconds; in the second group the specimens were ground on SiC papers, grits \#2400 and \#4000 for 60 seconds each, to ensure that the target surfaces of all beams were exactly in the same plane.

For in vitro toothbrushing a custom made abrasion testing machine (Tokyo Giken Inc., Tokyo, Japan) was used. The device is equipped with five lines of reciprocating toothbrushes and each line has a specimen holder $50 \mathrm{~mm}$ in length. Prospec Slim (GC Corp., Tokyo, Japan) toothbrushes (23 $\mathrm{mm}$ in length, 8 $\mathrm{mm}$ in width, medium hardness, $9.5 \mathrm{~mm}$ in filament length) were used. The holders with the ten composite specimens were mounted under the toothbrush heads with the lateral $1 \mathrm{~mm}$ wide specimen surfaces covered by a metal shield to protect these areas as reference planes from abrasion during testing. The toothpaste simulating abrasive was slurry of $150 \mathrm{~g}$ calcium

Table 1 Materials used

\begin{tabular}{|c|c|c|c|c|c|c|}
\hline Material/(code) & Type & Shade & Batch & Manufacturer & Composition* & $\begin{array}{c}\text { Filler content } \\
\text { wt } \% / \text { vol } \%\end{array}$ \\
\hline $\begin{array}{l}\text { Filtek Supreme } \\
\text { XT (FIL) }\end{array}$ & nanofill & A3B & $8 \mathrm{RB}$ & $\begin{array}{l}\text { 3M ESPE, St. Paul, } \\
\text { MN, USA }\end{array}$ & $\begin{array}{l}\text { Matrix: Bis-GMA, Bis- } \\
\text { PMA, TEGDMA, UDMA, } \\
\text { Filler: silica and zirconia } \\
\text { nanofiller }\end{array}$ & $78.5 / 59.5$ \\
\hline
\end{tabular}

$\begin{array}{llll}\begin{array}{l}\text { Grandio } \\ \text { (GRA) }\end{array} \text { nanohybrid } & \text { A3 } 0836152 & \begin{array}{l}\text { Voco, Cuxhaven, } \\ \text { Germany }\end{array}\end{array}$

Matrix: Bis-GMA, UDMA,

$87 / 71.4$ dimethacrylate, TEGDMA Filler: fluorosilicate glass, $\mathrm{SiO}_{2}$ microfiller, and spherical nanofiller

$\begin{array}{lll}\begin{array}{l}\text { Tetric EvoCeram nanohybrid A3 L28218 } \\ \text { (TET) }\end{array} & \text { Ivoclar Vivadent, } \\ & & \text { Schaan, } \\ & \text { Liechtenstein }\end{array}$

Matrix: Dimethacrylates

$82.5 / 68$ Filler: Ba-glass $1 \mu \mathrm{m}$ (48.5\%), Ba-Al-silicate glass $(0.4-0.7 \mu \mathrm{m}), \mathrm{YbF}_{3}$ (17\%) mixed oxide, prepolymers (34\%)

\begin{tabular}{|c|c|c|c|c|}
\hline $\begin{array}{l}\text { Venus Diamond } \\
\text { (VED) }\end{array}$ & nanohybrid & $\mathrm{A} 2$ & 010022 & $\begin{array}{l}\text { Heraeus Kulzer } \\
\text { GmbH, Hanau, } \\
\text { Germany }\end{array}$ \\
\hline
\end{tabular}

Matrix: TCD-DI-HEA, UDMA

Filler: Ba-Al-F-Borosilicate glass, $\mathrm{SiO}_{2}$ nanofiller

Bis-GMA: Bis-phenol-A-diglycidylmethacrylate, Bis-PMA: Bis-phenol-A-polyethyleneglycol diether dimethacrylate UDMA: urethane dimethacrylate, TEGDMA: triethylene glycol dimethacrylate, TCD-DI-HEA: 2-Propenoic acid, (octahydro-4,7methano-1H-indene-5-diyl) bis(methyleneiminocarbonyloxy-2,1-ethanediyl) ester.

*Composition as given by manufacturers 
carbonate (Calcium Carbonate 030-00385, Wako Pure Chemical Industries. Ltd., Osaka, Japan) dispensed in $100 \mathrm{ml}$ of water. According to the manufacturer the dried calcium carbonate powder has a purity of $99.5 \%$ (mass/mass), and the average particle size is $5.2 \mu \mathrm{m}$. The linear forth and back brushing action $(60 \mathrm{~Hz})$ on the specimens covering the entire length of $50 \mathrm{~mm}$ and immersed in the abrasive slurry was performed under $5 \mathrm{~N}$ load at ambient laboratory atmosphere for a total of 50,000 brushing cycles. The slurry was renewed for each test after each 10,000 cycles.

\section{Measurement of depth of wear and determination of surface roughness}

The surface roughness of each of the mounted specimens was determined with a profilometer (Surfcom 480A, Tokyo Seimitsu Co., LTD, Tokyo, Japan) equipped with a diamond pick-up (tip radius: 5 $\mu \mathrm{m}$, load: $4 \mathrm{mN}$ ). For determination of the abrasion depth the tracing length was $11 \mathrm{~mm}$ from the center of the one to the center of the opposite reference plane. For surface roughness $(4 \mathrm{~mm}$ tracing length in the center of each specimen) the stylus speed was $0.6 \mathrm{~mm} /$ second, and the cut-off $0.8 \mathrm{~mm}$. The average roughness $R \mathrm{a}$ of each specimen was measured close to the centerline of the $3 \mathrm{~mm}$ wide beams.

After each 10,000 brushing cycle the specimens were taken out of the slurry, rinsed under tap water, and gently air-dried for another surface roughness determination as described above. The depth of abrasion was determined graphically from the roughness registrations as the largest difference in $\mu \mathrm{m}$ between a line connecting the opposing reference planes and the deepest portion on the recorded profilometer trace.

Depth of wear and $R$ a values were analyzed by one-way ANOVA and Tukey's post-hoc test at the 5 percent level of significance. The wear data were additionally evaluated by linear regression analyses.

\section{Knoop Hardness measurements}

For each of the four resin composite materials 3 specimens were produced as above and polished on the top light activated surface with $\mathrm{SiC}$ paper, grit $\# 4000$ for 60 seconds. After 24 hours immersion in $37^{\circ} \mathrm{C}$ water Knoop hardness numbers $\left(\mathrm{kgf} / \mathrm{mm}^{2}\right)$ were determined from indentations made under $0.5 \mathrm{~N}$ load for 30 seconds at five locations close to the center of each specimen's top side (Hardness Tester HM-102, Akashi Co.,Yokohama, Japan). Statistical evaluation by oneway ANOVA and Tukey's post-hoc test $(p<0.05)$ was conducted.

\section{Scanning electron microscopy}

One random sample of each composite material and each pretreatment group after 50,000 brushing cycles was selected for SEM examination (Type VE-8800, Keyence Inc., Osaka, Japan). The samples were sputter-coated with Pt and photographs were taken of representative areas at $1,000 \times$ and $3,000 \times$ magnifications at $10 \mathrm{kV}$ acceleration voltage.

\section{RESULTS}

Figure 1 shows the relationships between abrasive wear for the four resin composites of samples pre ground with $\mathrm{SiC}$ paper \#600, simulating an initially worn, and $\mathrm{SiC}$ paper \#4000, simulating a perfectly polished restoration surface, respectively. The linear regressions were calculated on the base of the mean abrasion depths of 10 specimens each for the two different pretreatments, including the five measuring stages after 10,000 brushing consecutive cycles each. The circular signatures and whiskers are the mean depths of wear and the related standard deviations. All correlations were highly significant with coefficients of determination $\left(\mathrm{R}^{2}\right)$ between 0.984 and 0.995 . These pair-wise comparisons indicate that the surface conditions of the composites prior to testing had no direct impact on abrasive tooth brushing wear.

Figure 2 summarizes the wear depths of the composites tested by tooth brushing cycles. For each product wear increased linearly with the number of cycles. The bold and the stippled lines denote the linear regression line and the related 99 percent confidence intervals. The wear depths represent the pooled figures from the groups with different surface roughness at the start of the testing procedure, i.e. $n=20$ after each tooth brushing cycle. The wear of the nanohybrid material TET was between 7 and 40 times higher than the wear of the other three composites. When analyzed by one-way ANOVA the wear of the four resin composites after 50,000 brush cycles was significantly different $(p<0.001)$; Tukey's multiple comparison test proved differences between each of the composites $(p<0.05)$ with the following ranking order: GRA $<$ VED $<$ FIL $<$ TET.

Figure 3 depicts the Knoop hardness numbers of the tested composites with the highest values for GRA followed by the nanofill FIL, the nanohybrid VED and the nanohybrid TET with the filler-loaded prepolymerized particles. All groups were significantly different $(p<0.05)$.

Figure 4 shows the mean roughness $R$ a for each composite material measured before tooth brushing and after each brushing cycle with 10,000 strokes. Open bars refer to specimens, ground on $\mathrm{SiC} \# 600$, gray bars on \#4000. ANOVA proved highly significant differences between the groups for each material. The same lower and upper case letters in each graph denote statistically homogeneous groups, i.e. groups that were not significantly different $(p>0.05)$. TET exhibited the smallest $R$ a figures at all conditions tested. There was a slight but significant increase in $R$ a with increasing number of brushing cycles. The maximum roughness found for the nanofill FIL after 50,000 brushing cycles was less than $0.6 \mu \mathrm{m}$. In contrast, the glassfilled nanohybrid composites GRA and VED showed much higher roughness at each stage during abrasion testing. Although some groups of each material were found 

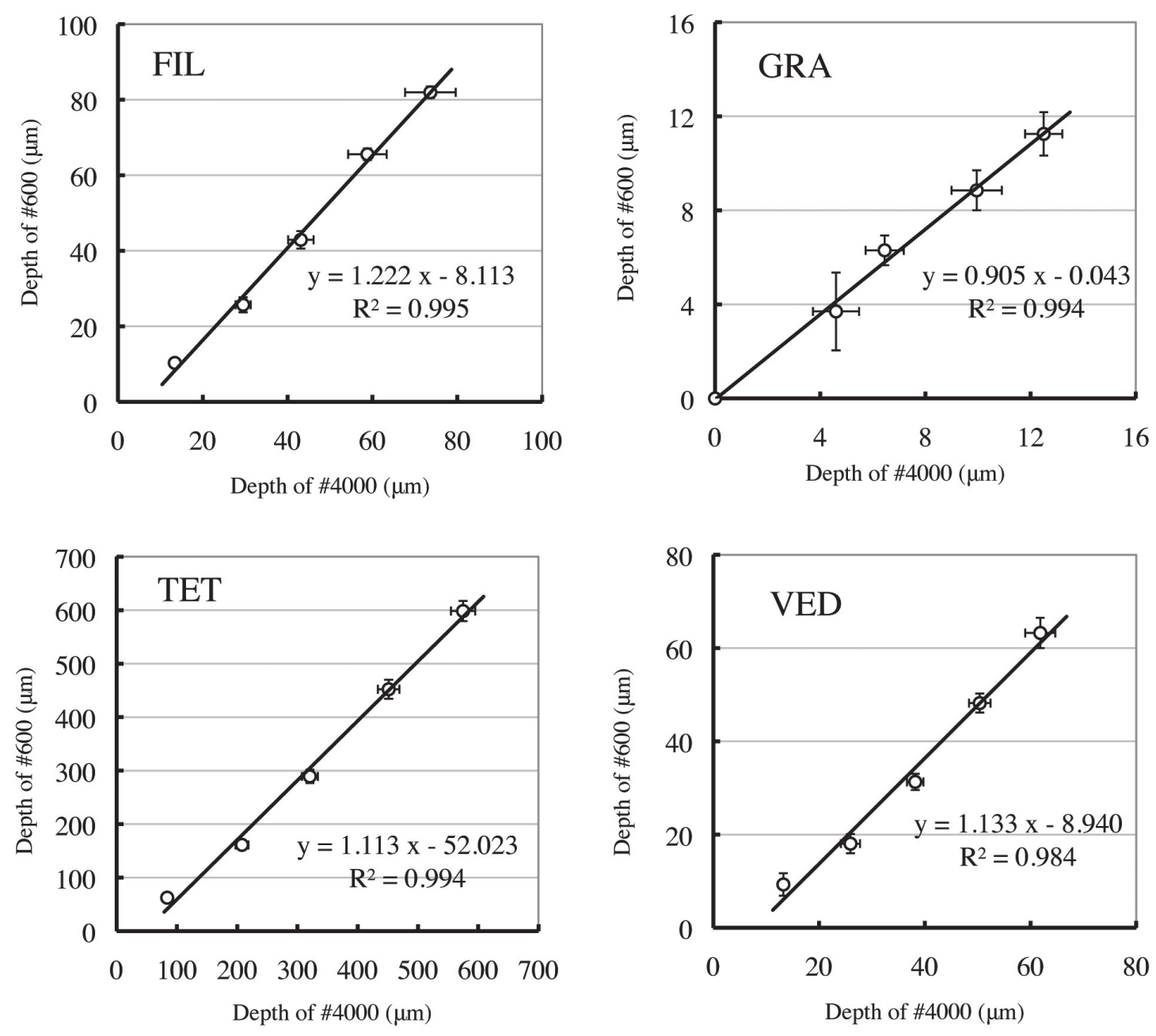

Fig. 1 Relationship between average depth of wear ( \pm standard deviations) after each 10,000 brushing cycle for FIL, GRA, TET and VED with initial surface roughness produced on SiC paper \#600 vs. $\mathrm{SiC}$ paper \#4000. Linear regression line and coefficient of determination $\left(\mathrm{R}^{2}\right)$.

with significantly different $R$ a figures at different brushing cycles there was generally not much difference in roughness between the five testing stages of specimens with different initial surface texture.

The textures of resin composite surfaces after 50,000 brushing cycles in calcium carbonate slurry are compared in Fig. 5. The SEMs were taken from specimens that were preground on $\mathrm{SiC}$ paper \#600. FIL shows densely packed, superficially abraded nanoclusters in the surrounding resin matrix. The surface is quite uniformly abraded. The surface of GRA that is characterized as nanohybrid material exhibits densely packed glass fillers with a wide grain size distribution. Most of the fillers with a flat ground surface are protruding from the surface while the matrix is selectively more abraded. In many locations the particle distance between neighboring glass fillers is less than $1 \mu \mathrm{m}$. TET is characterized by a very smooth and uniformly worn surface. There is no differential abrasion effect between the prepolymerized particles and the surrounding matrix. Small voids are seen throughout the entire surface. The appearance of the nanohybrid composite VEDs surface is very different from GRA. Large glass fillers, flat ground on the exposed surfaces, are clearly protruding from the surroundings. The distance between these larger particles is mostly wider than $10 \mu \mathrm{m}$.

Figure 6 illustrates the calcium carbonate particles used as abrasive agent. A shows a powder sample as delivered, whereas B shows the dried powder after use in the "toothpaste" slurry for 10,000 brushing cycles. Most of the crystalline species are rhombohedral and smaller than approximately $5 \mu \mathrm{m}$. The precipitated calcium carbonate powder shows typical cleavage that has resulted in prismatic, scalenohedral and tabular crystal species. Cleavage of primary particles into minor ones occurs under pressure exerted on the crystals, probably also during tooth brushing with the slurry used. 


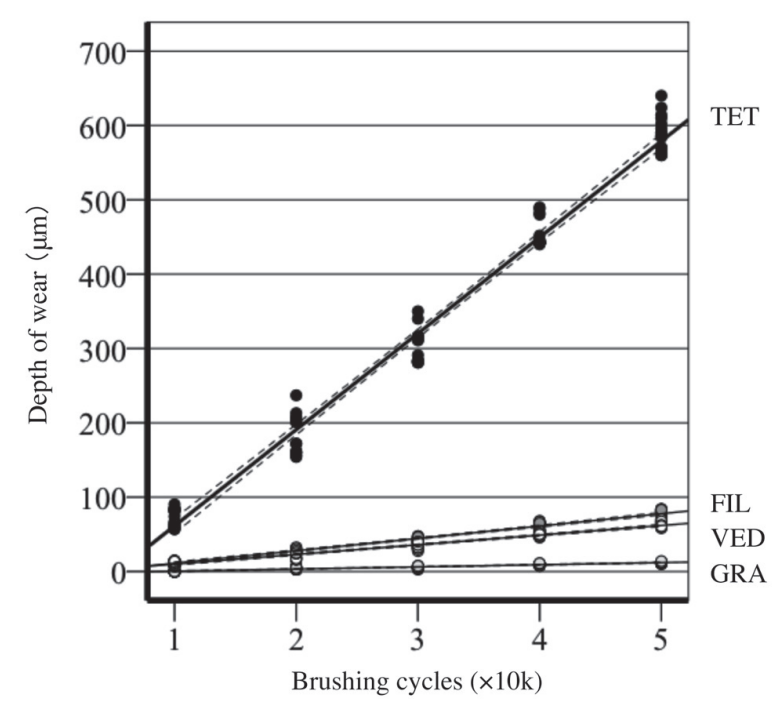

Fig. 2 Depths of wear of resin composites by brushing cycles. The linear regression lines and the 99 percent intervals of confidence (stippled lines) were calculated from the individual 100 readings each, including the two groups with different initial surface roughness. Coefficients of determination $\left(\mathrm{R}^{2}\right)$ were between 0.943 and 0.986 .

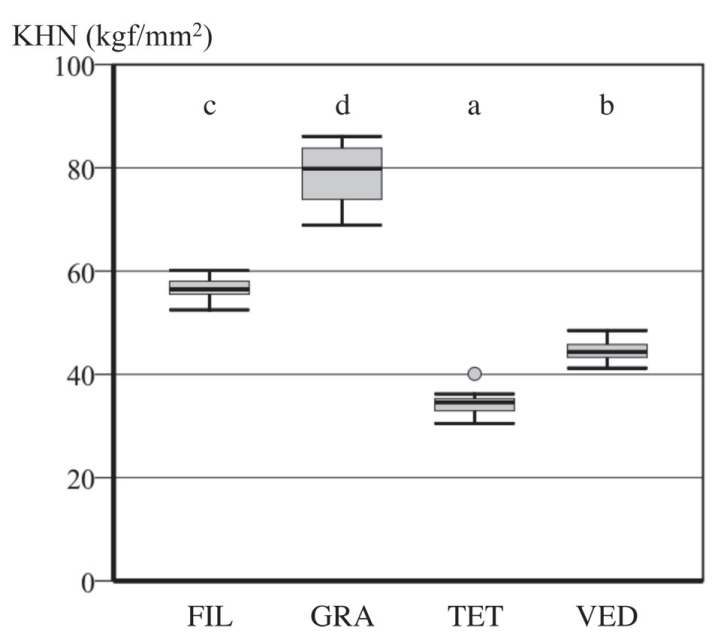

Fig. 3 Knoop hardness numbers (KHN) for the four resin composites after 24 hours storage in $37^{\circ} \mathrm{C}$ water. Box-and-whisker plots with medians and interquartile distances. Different lower-case letters denote groups that are significantly different $(p<0.05)$.

\section{$R \mathrm{a}(\mu \mathrm{m}) \quad$ FIL}

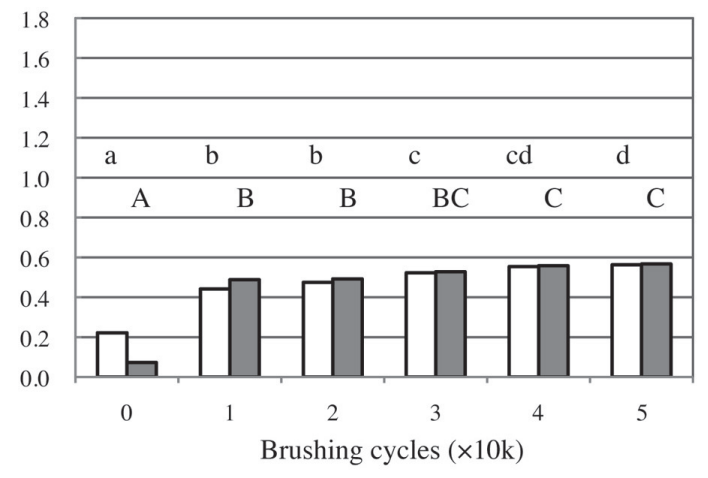

$R \mathrm{a}(\mu \mathrm{m}) \quad \mathrm{TET}$

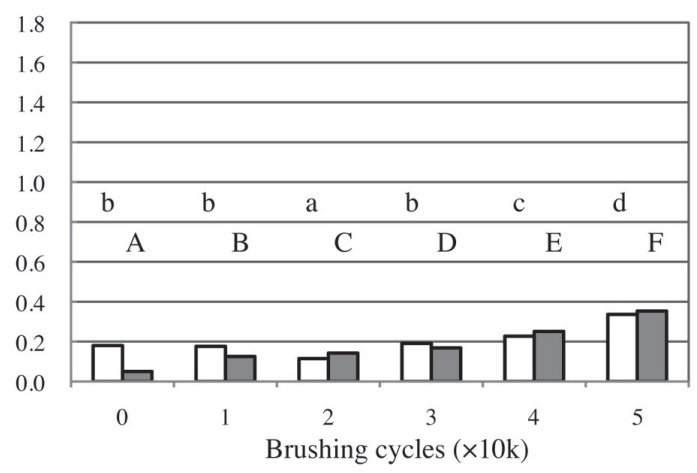

\section{$R a(\mu \mathrm{m}) \quad$ GRA}

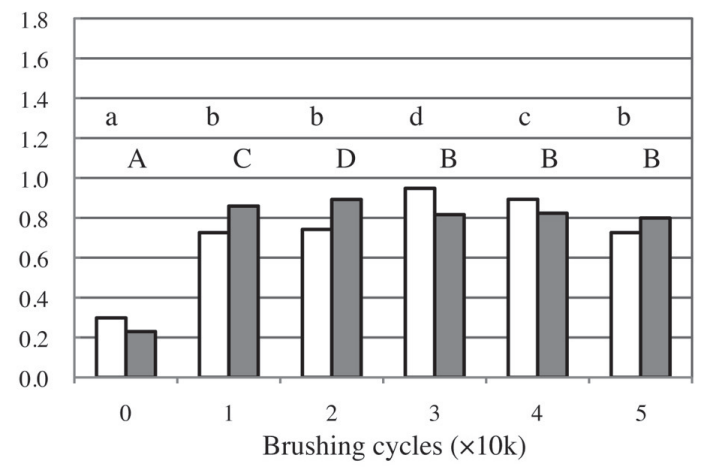

$R a(\mu \mathrm{m}) \quad$ VED

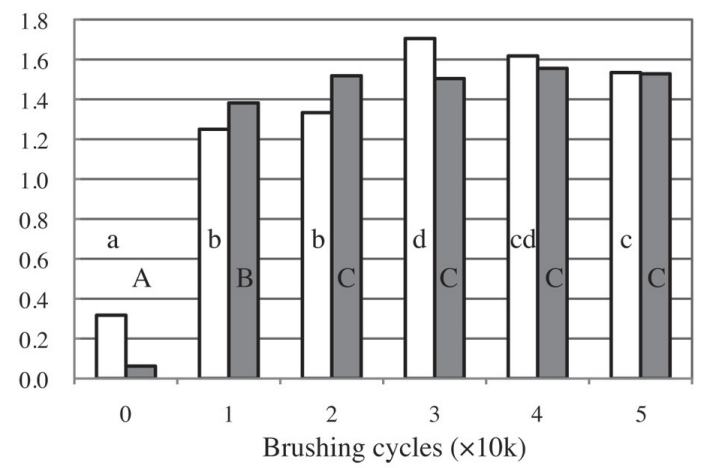

Fig. 4 Mean surface roughness (Ra) of FIL, GRA, TET and VED before brushing (0) and after brushing for 10,000 through 50,000 cycles. Open bars represent data from specimens ground with $\mathrm{SiC}$ paper \#600, gray bars with $\mathrm{SiC}$ \#4000. The same lower and upper case letters denote groups for the two different initial surface preparations, respectively that are not significantly different $(p>0.05)$. 

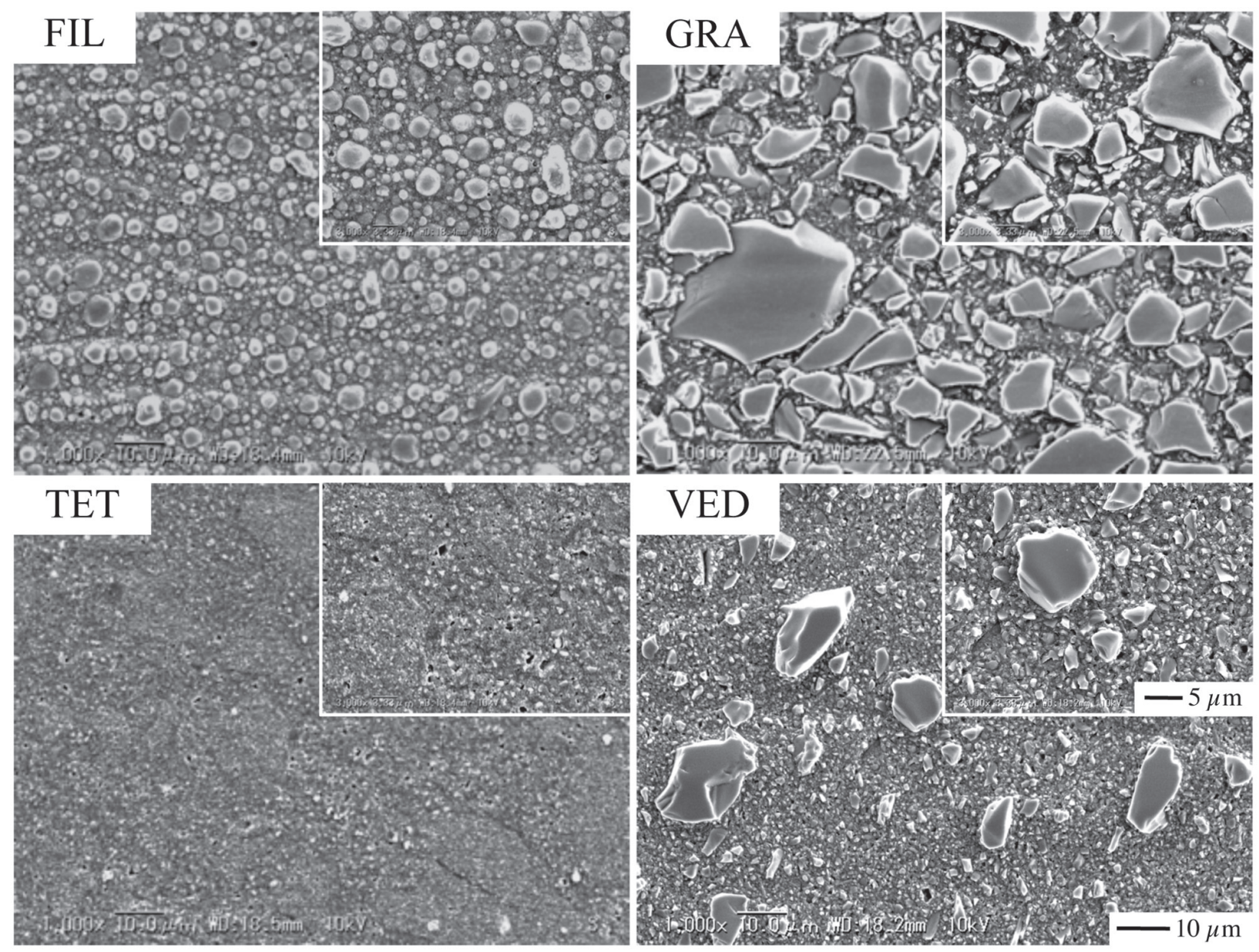

Fig. 5 Scanning electron microphotographs at $1 \mathrm{k}$ and $3 \mathrm{k}$ (insert) magnification of resin composite surfaces after 50,000 brushing cycles: FIL, GRA, TET and VED.
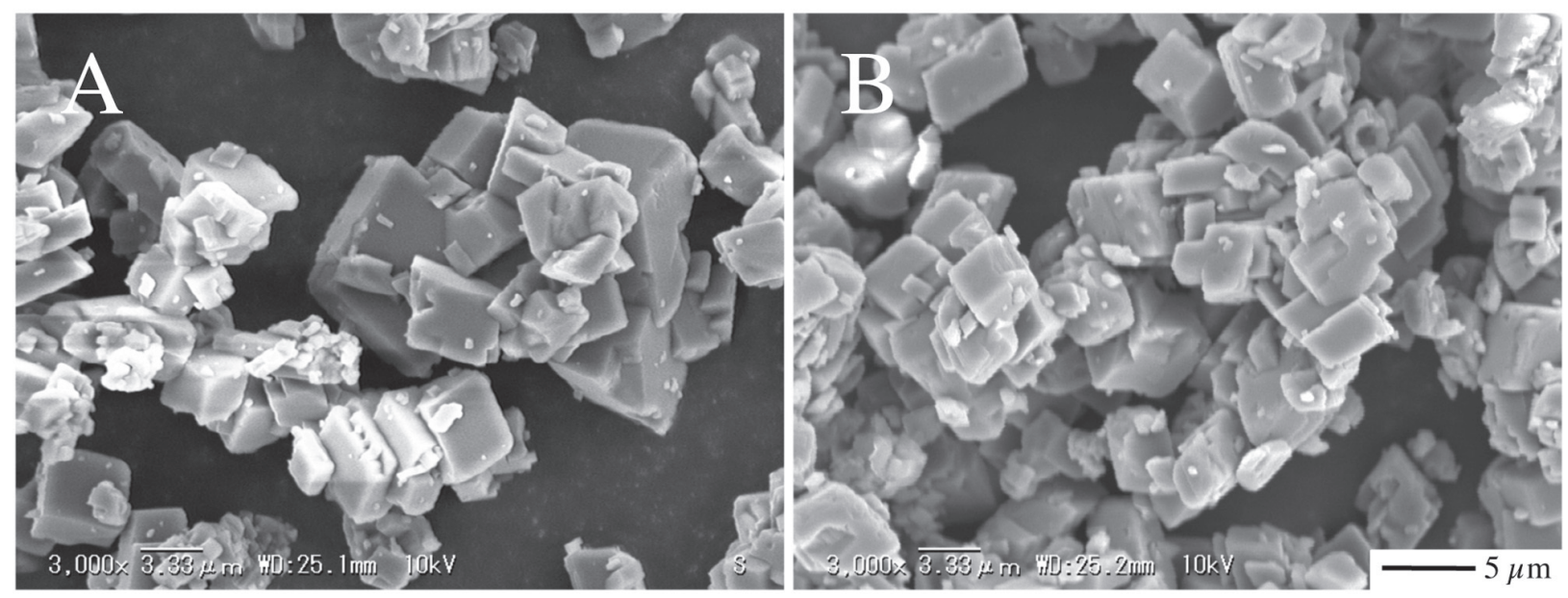

Fig. 6 Calcium carbonate powder of the PCC type (precipitated $\mathrm{CaCO}_{3}$ ) used as abrasive in the "toothpaste" slurry. A: dry powder; B: powder dried from slurry after 10,000 brushing cycles. The particles are mainly rhombohedral in shape, but also prismatic and tabular crystals are seen, as well as twinned crystals. 


\section{DISCUSSION}

A number of toothbrushing simulators have been proposed in literature with differences in fundamental design principles ${ }^{11,14,17)}$. However, so far no consensus has been reached on which design and parameter settings are most predictive for toothbrushing wear of resin composites.

In most of the published studies on toothbrushing abrasion of resin composites commercial toothpastes have been used. Our motivation to apply precipitated calcium carbonate as abrasive medium in aqueous slurry was to prevent other potential effects of commercial toothpaste formulations on the resin matrix of the resin composites used. Further, calcium carbonate is one of the frequently used abrasives in marketed toothpastes and a reasonably mild abrasive (Moh's hardness number 3). The abrasivity of calcium carbonate depends on the grain size, the smaller the particle the less abrasive it will be. In our test the abrasive particles had an average grain size of $5.2 \mu \mathrm{m}$.

Another issue is the type of toothbrushes to be used in simulators. We selected arbitrarily a medium hard type, frequently sold in Japan. Supposedly, the type of toothbrushes and bristle stiffness has scarcely any effect on resin composite wear. It has been documented that brushing of unfinished composite specimens with water had almost no effect on composite wear ${ }^{19)}$.

Concerning loading force of toothbrushes during action there is no standard either ${ }^{17,20-22)}$. In a clinical trial on 94 patients the average toothbrushing force registered was $350 \mathrm{~g}$ with a range from 140 through $720 \mathrm{~g}^{23)}$. We decided in agreement with Cho et al..$^{24)}$ and Senawongse and Pongprueksa ${ }^{18}$ to use a $500 \mathrm{~g}$ brushing force as a means to presumably accelerate wear in our simulator.

The number of toothbrushing cycles is another matter of dispute. It is assumed that 10,000 toothbrush strokes simulate approximately one year of toothbrush wear ${ }^{14,25,26)}$. In our study we applied 50,000 cycles in five consecutive runs with 10,000 cycles each, mainly because a resin composite like GRA showed very limited wear at fewer cycles. Variations of brushing speed have apparently no significant effect on wear ${ }^{17,20,27)}$. There is not much information available on the influence of simulated toothbrushing on resin composites after polishing with different systems ${ }^{15,28)}$. To elucidate this parameter in our trial we abraded composites that were either finished on SiC paper \#600 or polished on $\mathrm{SiC} \# 4000$. The results of the present trial showed that there is virtually no difference in wear depth or in surface roughness of the individual composites when the differently finished/polished specimens are subjected to toothbrush abrasion.

Wear of toothbrush-abraded specimens has been determined in different ways. Teixeira et al. ${ }^{29)}$ measured the difference in specimen thickness from their initial thickness using a micrometer caliper. Determination of specimen weight loss after being subjected to toothbrush abrasion is another method used $^{14,16)}$. This method has certain limitations when materials with high abrasion resistance and limited numbers of brushing strokes are investigated. Measurement of depth of wear with a computercontrolled three-dimensional measuring microscope and computing the volume loss from such data is proposed $^{30)}$. Due to the high resolution this method is apparently suitable to detect very small wear effects even when only few brushing strokes are used. In agreement with a previous study we decided to use a profilometric method to determine the depth of wear ${ }^{12}$. This method is advantageous as wear and roughness of the worn surface are determined consecutively with the same instrument.

In many published studies on resin composite wear, only surface roughness parameters or qualitative SEM examinations of the abraded composites are reported ${ }^{2,13,15,19)}$. Such an approach has however serious shortcomings, since assessment of surface texture as a single parameter disregards materials such as TET or GRA that showed low surface roughness and extremely high loss of substance or very little wear and quite high surface roughness in our study. In accordance with previous studies our trial confirmed that there was no positive correlation between wear and surface roughness ${ }^{29,311}$.

For understanding and interpretation of the present findings it was essential to determine both quantitative and qualitative wear data. The nanofill material FIL showed a moderate cumulative abrasion depth and rather uniform and smooth surface texture. The protruding nanocluster particles indicate that the wear mechanism follows the rule that wear starts with abrasion of the polymer matrix, followed subsequently by exposure of fillers, and finally removal of fillers. This process results in continuously increasing wear as demonstrated by the linear relationship between wear and number of brushing cycles. Dislodgment of nanoclusters from a nanofill composite has been reported previously ${ }^{32}$. Since the FIL clusters seen by SEM are all less than $2 \mu \mathrm{m}$ the wear process is comparatively slow.

The nanohybrid composite TET showed a very smooth and uniformly abraded surface without any discontinuity between the prepolymerized fillers and the surrounding matrix. The high wear rate found indicates that the product is mechanically relatively weak, as also demonstrated by the comparatively low Knoop Hardness, and thus easily abraded under the present test conditions. In contrast to a previous investigation we could not find any loss of prepolymerized particles ${ }^{18}$.

The data of the nanohybrid type composite materials GRA and VED look at first glance conflicting. Both products contain milled glass fillers next to the nanofiller loaded resin matrix. However, the wear, the surface roughness, and the Knoop hardness are very different. The filler loading of GRA is $71.4 \%$ (vol) and the glass particles are rather densely packed which is 
reflected by the Knoop hardness of around $80 \mathrm{kgf} / \mathrm{mm}^{2}$. This might be one of the reasons why the wear of this material is so small. The abrading calcium carbonate particles are probably too large, unless cleaved into very small parts, to enter into all inter particle matrix spaces for abrasion. Thus, the dense filler packing protects the matrix from fast abrasion and keeps the glass fillers longer retained in the matrix before being removed. The roughness of around $0.8 \mu \mathrm{m}$ after toothbrushing should be carefully interpreted. The tip radius of the profilometer used is $5 \mu \mathrm{m}$, and thus unable to dip down into the narrow spaces between many of the protruding glass filler particles to register the true roughness. Laser scanning profilometers, operating with beam diameters of around $1 \mu \mathrm{m}$, optical sensors or AFM will be able to record considerably more details and show higher roughness figures. This is one of the reasons why surface roughness figures from different studies cannot readily be compared. GRA resembles a more typical hybrid type resin composite than a nanohybrid type. The average particle size of 1 $\mu \mathrm{m}$ is misleading and the manufacturer should consider disclosing more details of the grain size distribution. The other nanohybrid resin composite, VED, has lower filler loading and more sparsely scattered large glass fillers than GRA. The Knoop hardness is only about 50 percent of the figure found with GRA. The abrasion is larger because the filled matrix area between the larger glass particles can readily be worn by the calcium carbonate abrasive. As a consequence, the protruding large glass particles will more easily lose grip from the matrix polymer and debond. The surface roughness measured was almost double the $R$ a value recorded for abraded GRA. This can only to some extent be explained by the stylus measurement method that is able to record more details on VED than on GRA.

The null hypothesis of this study that there would be no difference in toothbrushing wear, surface roughness and texture, and Knoop hardness between the four resin composites tested must be rejected.

Within the limitations of this study it can be concluded that toothbrushing abrasion of the four nanofiller resin composites results in significantly different wear rates and surface morphology. Regarding the manufacturers classification of these composites as universal types raises doubts whether the nanohybrid material Tetric EvoCeram with the comparatively low toothpaste abrasion resistance can fulfill this claim. Venus Diamond shows a reasonably moderate wear; however, VED develops high surface roughness during this toothbrushing procedure. Grandio shows very high abrasion resistance and a moderately high surface roughness caused by the calcium carbonate abrasive. The nanofill product Filtek Supreme XT with moderate abrasion and increase in roughness has apparently a good potential as a universal clinically successful resin composite.

\section{REFERENCES}

1) Ritter AV. Direct resin-based composites: current recommendations for optimal clinical results. Compend Contin Educ Dent 2005; 26: 481-492.

2) Jung M, Sehr K, Kliemek J. Surface texture of four nanofilled and one hybrid composite after finishing. Oper Dent 2007; 32: 45-52.

3) Ergücü Z, Türkün LS. Surface roughness of novel resin composites polished with one-step systems. Oper Dent 2007; 32: 185-192.

4) Moszner N, Salz U. New developments of polymeric dental composites. Prog Polym Sci 2001; 26: 535-576.

5) Mitra SB, Wu D, Holmes B. An application of nanotechnology in advanced dental materials. J Am Dent Assoc 2003; 134: 1382-1390.

6) Moszner N, Klapdohr S. Nanotechnology for dental composites. Int J Nanotechnol 2004; 1: 130-156.

7) Turssi CP, Saad JR, Duarte SL, Rodrigues AL. Composite surfaces after finishing and polishing techniques. Am J Dent 2000; 13: 136-138.

8) Yap AU, Tan CH, Chung SM. Wear behavior of new composite restoratives. Oper Dent 2004; 29: 269-274.

9) Turssi CP, Ferracane JL, Serra MC. Abrasive wear of resin composites as related to finishing and polishing procedures. Dent Mater 2005; 21: 641-648.

10) Chen MH, Chen CR, HSU SH, Sun SP, Su WF. Low shrinkage light curable nanocomposite for dental restorative material. Dent Mater 2006; 22: 138-145.

11) Heath JR, Wilson HJ. Abrasion of restorative materials by toothpaste. J Oral Rehabil 1976; 3: 121-138.

12) Momoi $\mathrm{Y}$, Hirosaki $\mathrm{K}$, Kohno $\mathrm{A}$, McCabe JF. In vitro toothbrush-dentifrice abrasion of resin-modified glass ionomers. Dent Mater 1997; 13: 82-88.

13) Neme AL, Wagner WC, Pink FE, Frazier KB. The effect of prophylactic polishing pastes and toothbrushing on the surface roughness of resin composite materials in vitro. Oper Dent 2003; 28: 808-815.

14) Wang L, Garcia FC, Amarante de Araujo P, Franco EB, Mondelli RF. Wear resistance of packable resin composites after simulated toothbrushing test. J Esthet Restor Dent 2004; 16: 303-314.

15) Heintze SD, Forjanic M. Surface roughness of different dental materials before and after simulated toothbrushing in vitro. Oper Dent 2005; 30: 617-626.

16) Prakki A, Cilli R, Amarante de Araujo P, Navarro MF, Mondelli J, Mondelli RF. Effect of toothbrushing abrasion on weight and surface roughness of $\mathrm{pH}$-cycled resin cements and indirect restorative materials. Quintessence Int 2007; 38: 544-554.

17) Parry J, Harrington E, Rees GD, McNab R, Smith AJ. Control of brushing variables for the in vitro assessment of toothpaste abrasivity using a novel laboratory model. J Dent 2008; 36: 117-124.

18) Senawongse P, Pongprueksa P. Surface roughness of nanofill and nanohybrid resin composites after polishing and brushing. J Esthet Restor Dent 2007; 19: 265-275.

19) Van Dijken JWV, Stadigh J, Meurman JH. Appearance of finished and unfinishes composite surfaces after toothbrushing. A scanning electron microscopy study. Acta Odontol Scand 1983; 41: 377-383.

20) Harte DB, Manly RS. Effect of toothbrush variables on wear of dentin produced by four abrasives. J Dent Res 1975; 54: 993-998.

21) Lewis R, Dwyer-Joyce RS, Pickles MJ. Interaction between toothbrushes and toothpaste abrasive particles in simulated tooth cleaning. Wear 2004; 257: 368-376.

22) Kon M, Kakuta K, Ogura H. Effects of occlusal and brushing forces on wear of composite resins. Dent Mater J 2006; 25: 
183-194.

23) Van der Weijden GA, Timmerman MF, Danser MM, van der Velden U. Relationship between the plaque removal efficacy of a manual toothbrush and brushing force. J Clin Periodontol 1998; 25: 413-416.

24) Cho LR, Yi YJ, Heo SJ. Effect of tooth brushing and thermal cycling on a surface change of ceromers finished with different methods. J Oral Rehabil 2002; 29: 816-822.

25) Goldstein GR, Lerner T. The effect of toothbrushing on a hybrid composite resin. J Prosthet Dent 1991; 66: 498-500.

26) Turssi CP, Hara AT, de Magalhaes CS, Serra MC, Rodrigues $\mathrm{AL} \mathrm{Jr}$. Influence of storage regime prior to abrasion on surface topography of restorative materials. J Biomed Mater Res 2003; 65: 227-232.

27) Harte DB, Manly RS. Four variables affecting magnitude of dentifrice abrasiveness. J Dent Res 1976; 55: 322-327.
28) Neme AL, Frazier KB, Roeder LB, Debner TL. Effect of prophylactic polishing protocols on the surface roughness of esthetic restorative materials. Oper Dent 2002; 27: 50-58.

29) Teixeira ECN, Thompson JL, Piascik JR, Thompson JY. In vitro toothbrush-dentifrice abrasion of two restorative composites. J Esthet Restor Dent 2005; 17: 172-182.

30) Wonglamsam A, Kakuta K, Ogura H. Effects of occlusal and brushing cycles on wear of composite resins in combined wear test. Dent Mater J 2008; 27: 243-250.

31) Tanoue N, Matsumura H, Atsuta M. Analysis of composite type and different sources of polymerization light on in vitro toothbrush/dentifrice abrasion resistance. J Dent 2000; 28: 355-359.

32) Yap AU, Yap SH, Teo CK, Ng JJ. Comparison of surface finish of new aesthetic restorative materials. Oper Dent 2004; 29: 100-104. 